\title{
Application of NFC Technology and Short Distance Wireless Communication Technology in Intelligent Meter Readling
}

\author{
Gu Jiang Tao, Wang Ying, Sun Jing, Xu Ying \\ Anhui Nanrui Zhongtian Power Electronics Co., Ltd., Hefei 230011, China
}

\begin{abstract}
With the popularization of smart phones and the continuous popularization of NFC near field communication technology, low-power short-range wireless communication technology is widely used in wireless meter reading. It is used for meter reading and compatibility in existing systems. This paper de-signs a wireless meter reading system based on short distance wireless communication technology and NFC. The system is stable, low cost and good versa-tility. The system is based on short distance wireless communication technology and NFC wireless meter reading system.
\end{abstract}

Key words: NFC; wireless meter reading; short dis-tance wireless communication

Published Online: $30^{\text {th }}$ Sept 2017

\section{Introduction}

With the rapid increase in the level of information technology applications, the domestic public utility metering meter reading method has been developed into a wireless meter reading from manual copy, es-pecially micro-power wireless technology in the wire-less meter reading of the application, so that meter reading becomes more accurate and timely. With the popularity of smart phones and NFC near field com-munication technology continues to promote, making people's lifestyles become more convenient and col-orful.

NFC technology evolved from contactless radio fre-quency identification (RFID), developed by Philips Semiconductors (now NXP Semiconductors), Nokia and Sony, based on RFID and interconnect technol-ogies. Near Field Communication (NFC) is a short-range high-frequency radio technology, running at $13.56 \mathrm{MHz}$ frequency of $20 \mathrm{~cm}$ distance. The trans-mission speed of $106 \mathrm{Kbit} / \mathrm{s}, 212 \mathrm{Kbit} / \mathrm{s}$ or 424 $\mathrm{Kbit} / \mathrm{s}$ three. At present, near field communication has been adopted as ISO/IEC IS 18092 international standard, ECMA-340 standard and ETSI TS 102 190 standards. NFC uses both active and passive read modes.

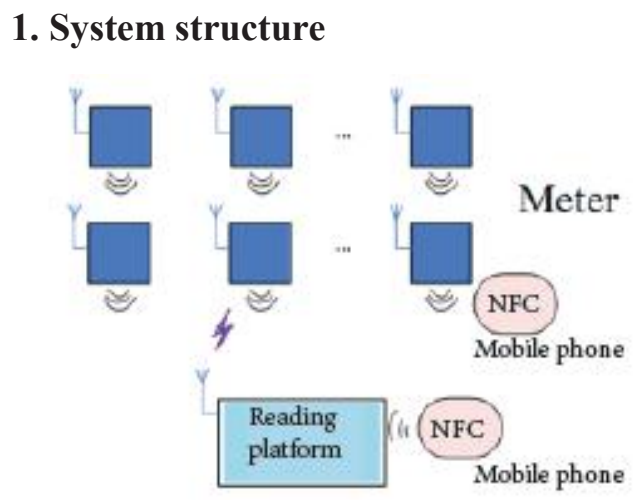

Figure 1 System block diagram

As shown in Figure 1, the system has a meter, reading platform and support NFC mobile phone components. The system supports the meter side NFC mobile phone software meter reading and reading platform side NFC mobile phone software meter reading in two modes, which is convenient for users to get their own usage in different situation, but also to facilitate the meter reading staff to conduct a comprehensive meter reading.

There are two ways to read the system, the method 1: the user directly read the meter through the NFC phone with the embedded module interaction, em-bedded NFC module through the MCU data will be sent to the industry meter, and then the meter back to the data through the NFC back to the NFC phone to complete the meter reading function. Method 2: through the reading platform of NFC mobile phone to complete the meter reading. The NFC module from the NFC mobile phone and reading platform will be copied to the reading platform to read the command which will be 
resolved through the short-range wireless communication technology. After that, the order is sent to the industry meter, and then the data returned by the industry meter is returned to the reading platform through the short-range wireless communication technology. The data is unpacked by the reading platform and the data is returned to the mobile phone through the NFC mod-ule after the packet is reassembled to complete the meter reading function.

\section{System hardware structure design}

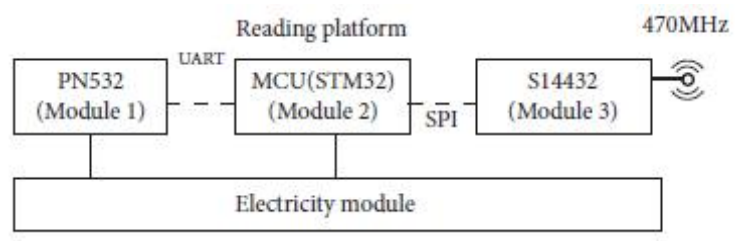

Figure 2 Reading platform hardware structure

As shown in Figure 2, the reading platform is mainly composed of PN532 module, MCU main control module, SI4432 RF module and power module, in which PN532 is introduced by NXP to support NFC protocol RF chip, the use of NFC-DEP protocol for data exchange; MCU main control module ST STMicroelectronics STM32F4XX series of chips, the chip supports DSP high-performance chips to support low-power mode of operation; SI4432 for the core laboratory launched a micro-power chip. This invention uses the free license ISM band 470Mhz communication, the use of GFSK modulation with good penetration and anti-jamming capability, because most of the time the whole system work in low power mode, so the power supply module can be the rechargeable battery for long term work.

PN532 module and the main control module MCU com-munication between the use of UART protocol, the baud rate is set to $115200 \mathrm{bps}$, wireless communication module SI4432 and MCU through the SPI interface to connect the communication, the RF antenna with a gain of $3.5 \mathrm{dBi}$ sucker antenna

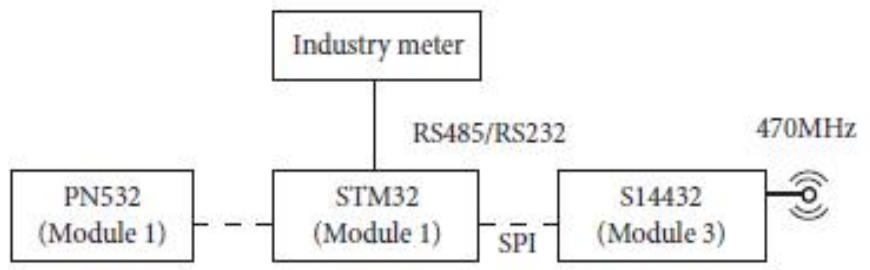

Support NFC mobile phone

Figure 3 Embedded wireless module composition dia-gram

Figure 3 for the embedded wireless module, the module embedded in the industry meter, powered by the indus-try meter, the module mainly by the main control module MCU and RF SI4432 module. MCU main control mod-ule ST STMicroelectronics STM32F4XX series chip, the chip supports DSP high-performance chip to support low-power mode of operation; SI4432 for the core labo-ratory launched a micro-power chip, the invention uses a license-free ISM Frequency band 470Mhz communica-tion, using GFSK modulation with good penetration and anti-jamming capability.

Among them, the main control module MCU and the industry between the use of industry standard interface RS485 or RS232 connection communication, wireless communication module SI4432 and MCU through the SPI interface to connect the communication, the RF an-tenna with a gain of $3.5 \mathrm{dBi}$ sucker antenna.

\section{System function to achieve}

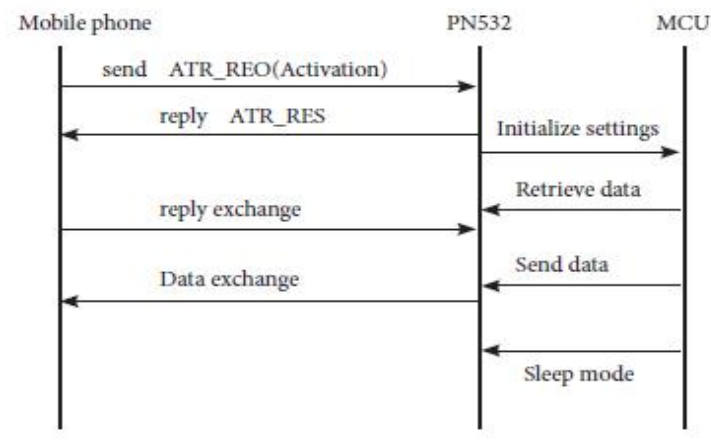

Figure 4 Interactive flow chart

Figure 4 is the interactive process between the instrument (support NFC mobile phone) and the reading platform/ meter side NFC module. The procedure is as follows:

In step $\mathrm{S} 1$, the mobile phone opens the NFC switch and the meter reading client (the meter reading software is not within the scope of the invention), selects the meter reading instruction, and activates the NFC module of the reading platform near the NFC tag antenna of the reading platform.

In step S2, the NFC module whose read platform is active is locally initialized according to the received informa-tion. The NFC protocol is NFC-DEP and the rate is $212 \mathrm{Kbps}$.

In step S3, the PN532 is set to the receiving mode, the communication command issued by the mobile client is obtained, and the command is transmitted to the 470Mhz micropower network.

Step S4, the 470 network upload data package, set the PN532 for the send mode, the data uploaded to the mo-bile client.

Step S5, after the completion of data exchange, the PN532 is set to sleep mode. 
Step S6, and other data are exchanged, and steps S1 to S5 are repeated.

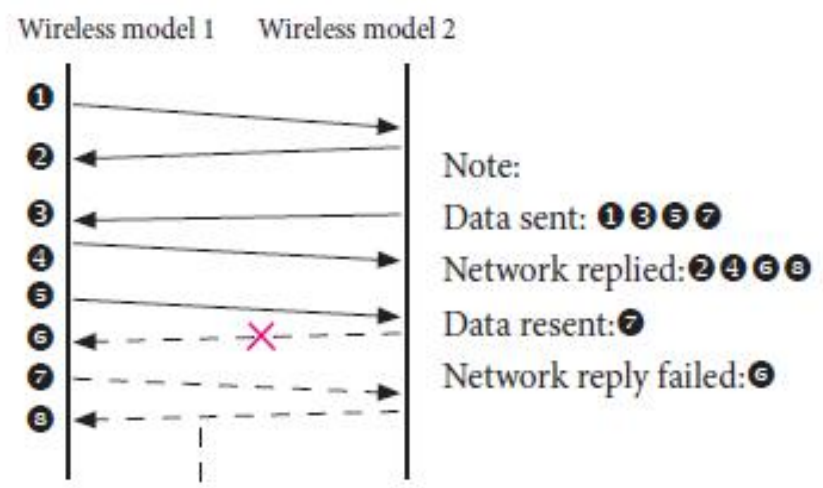

Figure 5 Wireless data commucation flow chart

Figure 5 shows a schematic diagram of the data commu-nication flow of the micropower 470 network, assuming that there is already a route between the reading platform and the embedded wireless module. The data communi-cation process is as follows:

In step $\mathrm{S} 1$, the wireless module 1 transmits data to the wireless module 2 .

In step $\mathrm{S} 2$, after receiving the data, the wireless module 2 judges whether the data is valid or not. If the network module is successfully received, the wireless module 2 does not reply to the network reply if it receives the data.

In steps $\mathrm{S} 3$ to $\mathrm{S} 4$, the wireless module 2 transmits data to the module 1, and steps S1 to S2 are performed.

In step S5, the wireless module 1 transmits data to the wireless module 2 .

In step S6, the wireless module 1 fails to successfully receive the network reply due to the signal difference or the like.

In step S7, after the wireless module 1 waits for a trans-mission timeout, the wireless module 1 resends the data to the wireless module 2 .

\section{Mobile terminal application implementation}

\subsection{Overall structure}

The application is based on ECLIPSE + ANDROID SDT Android development platform to developed, in which divided into Android mobile terminal APP program, Web server interface program, Web server background man-agement program, the three system programs to support. Mobile APP program using cordova2.9 framework, based on HTML5 and CSS3 standard Bootstrap framework for interface layout. Web server interface using the standard jsonp interactive mode, the background program using java language development, background framework us-ing spring mvc3.0, the database using lightweight Sql-Lite. Web server background management said Zeng $\mathrm{Xu}$ Bootstrap interface design, the background program us-ing java language development, background framework using spring mvc3.0, the database using lightweight Sql-Lite.

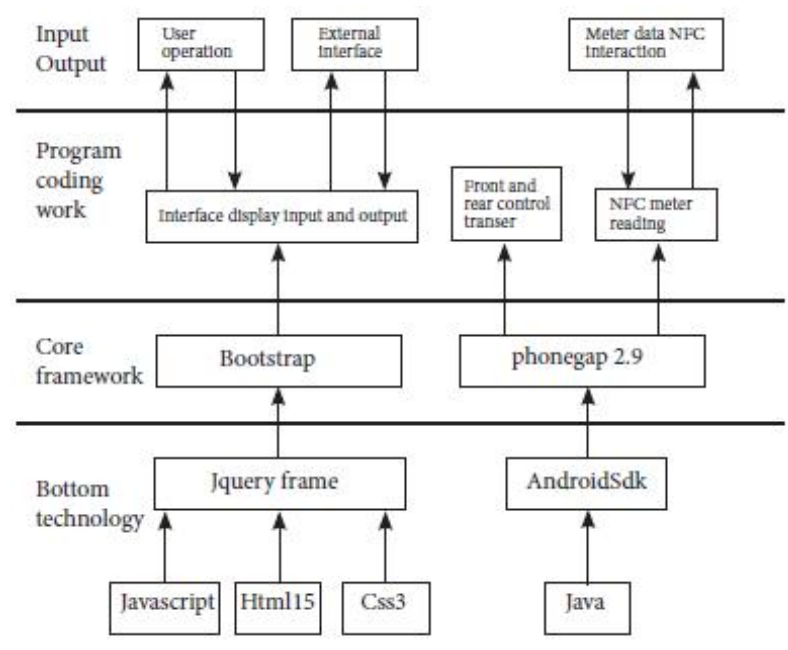

\subsection{Main functions}

The mobile application operation function consists of the following functions:

\section{- Register}

The first time to open the application, will display the user registration interface, the user clicks on registration, verify the account number, password, second password, cell phone number and other information is empty, if empty is prompted to re-enter, if verified, the data en-cryption will upload to the web interface server, the web interface server receives the data to judge and return the prompt message.

The message is as follows:

1) Please wait for the administrator to review (the basic data in the number and telephone number does not match, by the manual audit).

2) The account number cannot be registered (no data is available in the base data).

3) Please sign in successfully.

- Login

User login interface, the user login information encryp-tion, through the jsonp interactive way to the Web inter-face program, the interface program to determine the re-turn on the user login success or failure flag, if the login fails to return to failure information, the failure of the fol-lowing information:

1) No user information.

2) The user is not registered (the database exists 
user in-formation, you can log on after registration).

3) Incorrect password please re-enter (user password in-put is incorrect).

4) The Web server cannot respond.

5) User information is being reviewed, please later (al-ready registered, but the administrator failed to review).

6) User information audit does not pass, please contact the administrator (already registered, but the administra-tor will lock the user cannot log on).

- User information

After landing the user information interface, in the login interface, the web server according to the user name and password to judge the user's basic information through the mobile app decrypt which displayed in the user infor-mation interface.

\section{- Power collection}

Power to read the interface, click copy power data, the interface into the NFC interface; this time the phone near the NFC meter, will send a copy of the message, order the meter to return to 7 days before all the electricity data. Af-ter the data is returned, the copy power will be displayed after the value. If the NFC matches or the meter returns a message error, the failure prompt is displayed. Data cop-ied back, it will be stored in the app comes with the da-tabase, the next login, but also to see the last copy of the data, when the click to clear the historical data, save the data disappeared.

\section{- Other information copy}

Data reading module can read other meter readable infor-mation, such as rated current, rated voltage, table number and so on. Click on the copy appears NFC match inter-face, the phone close to the meter, read the meter data, meter data back, the copy value will be displayed in the interface. Other data read, is configurable data, when the user login, the web interface will return the user can copy items, if the administrator can be set to empty the user is empty, the interface is empty, which cannot copy any meter readable data.

\section{Conclusion}

The system uses $470 \mathrm{MHz}$ (ISM band) wireless commu-nication system and NFC near the field communication technology dual system structure, the whole system in-cludes embedded in the meter in the embedded wireless module, reading platform (gateway module, complete 470 networks with the NFC Data conversion), support for NFC phones. $470 \mathrm{Mhz}$ wireless communication system through the mesh ad-hoc network technology, can auto-matically select the optimal link, with ad hoc network, automatic repair network function to improve the network reliability and anti-jamming capability for wireless me-ter reading technology development and application have played a positive role in the promotion.

\section{References}

[1] Yu Wen Bo. NFC Technology Based on the Applica-tion of Intelligent Terminals. China's New Communica-tion, 2013, (7).

[2] Zhang $\mathrm{Su} \mathrm{Na}$, Jia Li Jun, Zhang Huai $\mathrm{Wu}$, Wang Chi Jun, NFC Mobile Phone Research Progress. Communica-tion Technology, 2012, (12).

[3] Wang Jian Ke. NFC in the Application of Intelligent Terminals. China's New Communications, 2016, (3).

[4] Xie Xi Dong, Yang Yi, Han Fang, Yang Yan Zhong, Xu Pei Pei, College of Information Science and Technol-ogy, Nanjing University of Aeronautics and Astronautics.

[5] Zhu Zheng Jian, Xiao Zhi Hua, Design and Imple-mentation of Electricity Mobile Payment System Based on NFC. Electrical Measurement and Instrumentation, 2013, (6).

[6] Guo Liang, Si4432-based Radio Frequency Transceiv-er System Design. SCM and Embedded Systems Applica-tions. 2009, (11).

[7] Chen Jun Jie, Zhu Zhi Jie, Wen Yao Jun, PN532-Based Contact and Non-Contact Card Reader Design. Electronic Technology Application, 2010, (5).

[8] Zhang Hai Feng, Zhang Pei, Design of RFID Reader

Based on PN532. Journal of Hangzhou Dianzi University, 2012, (6).

[9] Zhu Liu, Stm32-Based Non-Contact Reader Design. Silicon Valley, 2014, (19).

[10] Pan Xubing, Design of Wireless Transceiver Mod-ule Based on Si4432. Journal of Computer Applications, 2009, (12).

[11] Hu Zhong Wang, Mo Jia Qing, Mobile Application Development Based on Android. Software Guide, 2014, (11).

[12] Su Han Jiang, HTML5-Based Mobile Application Development Research. Network security Technology and Application, 2014, (11). 